\title{
INTERLACING OF EIGENVALUES AND INVARIANT FACTORS
}

\author{
J. F. Queiró, E. M. SÁ, A. P. Santana and O. Azenhas
}

Abstract. In this note, we explain the analogy between interlacing properties of invariant factors of matrices over a principal ideal domain $R$ and of eigenvalues of complex Hermitian matrices. This is done by looking at the interlacing theorems as dealing with very special cases of the problems of describing the invariant factors of a product of $R$-matrices and the eigenvalues of a sum of Hermitian matrices.

Mathematics subject classification (1991): 15A42.

Key words and phrases: Eigenvalues, invariant factors, interlacing.

\section{REFERENCES}

[1] D. Carlson, Minimax and interlacing theorems for matrices, Linear Algebra and its Applications 54 (1983), 153-172.

[2] D. Carlson and E. Marques de Sá, Generalized minimax and interlacing theorems, Linear and Multilinear Algebra 15 (1984), 77-103.

[3] Ky Fan and G. Pall, Imbedding conditions for Hermitian and normal matrices, Canad. J. Math. 9 (1957), 298-304.

[4] I. Gohberg and M. A. Kaashoek, Unsolved problems in matrix and operator theory, II. Partial multiplicities for products, Integral Equat. and Oper. Theory 2 (1979), 116-120.

[5] G. J. Heckman, Projections of orbits and asymptotic behavior of multiplicities for compact connected Lie groups, Invent. Math. 67 (1982), 333-356.

[6] T. Klein, The multiplication of Schur functions and extensions of $p$-modules, J. London Math. Soc. 43 (1968), 280-284.

[7] A. Klyachko, Stable bundles, representation theory and hermitian operators, Selecta Mathematica 4 (1998), 419-445.

[8] A. Knutson and T. Tao, The honeycomb model of $G L_{n}(\mathbb{C})$ products I: Proof of the saturation conjecture, J. Amer. Math. Soc. 12 (1999), 1055-1090.

[9] I. G. MacDonald, Symmetric Functions and Hall Polynomials, Oxford University Press, Oxford, 1995.

[10] J. F. Queiró, Generalized approximation numbers, Portugaliae Mathematica 43 (1985/86), 157-166. Correction, ibidem, 45 (1988), 59.

[11] E. Marques de Sá, Imbedding conditions for $\lambda$-matrices, Linear Algebra and its Applications 24 (1979), 33-50.

[12] E. Marques de Sá, Interlacing Problems for Invariant Factors (English version of a Portuguese unpublished draft of 1978), Textos de Matemática - Série B, Dep. Matemática, Univ. Coimbra, 1998.

[13] A. P. Santana, J. F. Queiró and E. Marques de Sá, Group representations and matrix spectral problems, Linear and Multilinear Algebra 46 (1999), 1-23.

[14] A. Schrijver, Theory of Linear and Integer Programming, John Wiley, Chichester, 1986.

[15] R. C. Thompson, The behavior of eigenvalues and singular values under perturbations of restricted rank, Linear Algebra and its Applications 13 (1976), 69-78.

[16] R. C. Thompson, Interlacing inequalities for invariant factors, Linear Algebra and its Applications 24 (1979), 1-32.

[17] H. Wielandt, Topics in the Analytic Theory of Matrices, Lecture Notes, Univ. Wisconsin, 1967. 